\title{
lubricants
}

ISSN 2075-4442

www.mdpi.com/journal/lubricants

Article

\section{Influence of Base Oil Polarity on the Transient Shear Flow of Biodegradable Lubricating Greases}

\section{Martin Fiedler ${ }^{1}$, Rubén Sánchez ${ }^{2}$, Concepción Valencia ${ }^{2}$, Claudia S. Leopold ${ }^{3}$, Erik Kuhn ${ }^{4}$ and José M. Franco ${ }^{2, *}$}

1 Fuchs Schmierstoffe GmbH, Friesenheimer Straße 19, 68169 Mannheim, Germany;

E-Mail: martin.fiedler@fuchs-schmierstoffe.de

2 Complex Fluid Engineering Laboratory, Department of Chemical Engineering,

Faculty of Experimental Sciences, University of Huelva, Huelva 21071, Spain;

E-Mails: ruben.sanchez@diq.uhu.es (R.S.); barragan@uhu.es (C.V.)

3 Pharmaceutical Technology, Institute of Pharmacy, University of Hamburg, Bundesstr 45,

Hamburg 20146, Germany; E-Mail: Claudia.Leopold@uni-hamburg.de

4 Laboratory of Machine Elements and Tribology, Department of Mechanical Engineering and Production, Hamburg University of Applied Sciences, Berliner Tor 5, Hamburg 20099, Germany; E-Mail: Erik.Kuhn@haw-hamburg.de

* Author to whom correspondence should be addressed; E-Mail: franco@uhu.es;

Tel.: +34-959219995.

Academic Editor: James Krzanowski

Received: 1 July 2015 / Accepted: 31 August 2015 / Published: 9 September 2015

\begin{abstract}
The scope of this study is to elucidate the physical mechanisms influencing the transient flow behavior of lubricating greases based on biogenic oleochemicals from a polarity point of view. This includes the mutually interacting influence of base oil polarity and thickening agents on the rheologically-measured mechanical structural degradation in transient shear flow. Due to the high temperature dependence of Keesom forces in the background of polar-active bond mechanisms, the analysis of the transient flow response as a function of temperature allows to attribute the observed influences to differences in base oil polarity. In general, clay-thickened greases show a greater tendency to be rheologically influenced by base oil polarities than soap-thickened lubricating greases.
\end{abstract}

Keywords: biodegradable lubricating greases; AFM; oil polarity; rheology; transient flow 


\section{Introduction}

Lubricating greases mainly consist of a base oil and a thickening agent of either fibrous, platelet or other aggregate structure in which the physically-acting bond forces build a stable three-dimensional network. This network has the inherent ability to regain the structure after mechanical stress. Thus, greases are known to exhibit both solid and liquid state properties. In a lubricated contact, the viscoelastic properties of greases, along with their tackiness, allow them to remain in a spot that can be tribologically stressed, even repeatedly. On the other hand, their flow properties allow greases to yield deformational stress evoked by the relative motion of sliding or rolling contacting partners while still serving as an intermediate medium separating the two contacting surfaces on the micro scale. These characteristics have qualified greases to be the lubricants of choice in a variety of applications, foremost led by rolling bearings [1,2]. These characteristics also physically define greases as non-Newtonian liquids. Large effort has been undertaken in the past and present research to rheologically investigate lubricating greases [3-7].

Lately, the growing awareness of ecological issues in legislation, as well as customer responsibility, has led to a growing demand of environmentally-friendly lubricants. According to Wilson [8], a huge amount of lubricant leaks into the ground and sea in industrial operations leading to pollution of the environment. In the last decades, biological greases have been introduced into a variety of industrial and consumer applications in order to protect the environment from the negative effects of lubricants based on petrochemically-refined mineral oils. As a simple, yet effective, approach to bio-lubricating grease design in many cases the traditional base oils have been substituted by rapidly biodegradable oleochemicals - mostly refined from vegetable oils. As vegetable-derived oleochemicals intrinsically exhibit positive tribological properties, these substituted greases must not inevitably be inferior to the original greases based on mineral oils. This is substantiated by physisorptive interaction of these oleochemicals with metallic tribological surfaces. However, in additized greases, oleochemicals may compete with some additives on surface adsorption leading to higher wear rates [9]. Biologically rapidly-degradable oils, especially esters of glycerol with saturated and unsaturated long chain fatty acids, are known to exhibit very high relative polarities, which enable these adsorption processes by van der Waals bonds [10,11]. The mechanisms of van der Waals forces rest upon electrostatic interaction between molecules with permanent or temporary dipoles [12], resulting either in Keesom, London or Debye forces, depending on the dipole character. While Keesom forces are the strongest secondary valence bonds, London and Debye forces only play a minor role in the scope of this study. The O-C bonds of esters and the O-H bonds of fatty acids and alcohols in oleochemicals evoke a large charge imbalance caused by different electronegativities. This results in the formation of permanent dipoles [13], high tendencies towards the formation of Keesom forces and consequently high polarities of oleochemicals. In recent studies it has been proven that high base oil polarities, caused by the use of biodegradable oleochemicals in lubricating greases, may heavily influence their tribological characteristics due to surface adhesion processes in tribo-contacts, as shown in [14]. The polarity-induced influence described therein gives reason to presume that base oil polarities not only affect the tribological but also the rheological characteristics of lubricating greases. Therefore, it has been proposed to examine the transient flow behavior of greases in dependence of the base oil polarity in further investigations. Rheological investigations of lubricating greases have numerously been 
performed; some even detected polarity influences in amplitude-sweep tests $[15,16]$. The main contribution of this study is the exclusive focus on the influence of base oil polarity on the rheologically-measured shear-induced structural breakdown of biodegradable greases.

\section{Experimental Section}

\subsection{Lubricating Grease Samples}

Several greases, soap and clay-thickened, were rheologically investigated in the course of this study. Three biodegradable and biogenic organic esters were used as base oils to formulate the investigated lubricating greases: high-oleic sunflower oil (HOSO), octyldodecyl isostearate (OCT), and trimethylolpropane trioleate (TMPO). For the sake of comparison with a well-known synthetic grease system, a biodegradable low viscous polyalpha olefin (PAO) was used as reference base oil in the formulation process. Lithium and calcium 12-hydroxystearate were used as metallic soap thickeners and quaternized bentonite (BT) and a highly-dispersed silica acid (HDS) were used as mineral clay thickener agents. No additives were included in the formulation except for a swelling aid in the case of the clay-thickened greases. Composition and characteristics of the greases are displayed in Table 1.

Table 1. Grease composition and some physical characteristics.

\begin{tabular}{c|c|c|cccc}
\hline Grease & \multicolumn{2}{|c|}{ Thickener } & Swelling & Pu $^{\text {a }}$ & Pw $^{\mathbf{a}}$ & NLGI $^{\mathbf{b}}$ \\
\hline Label & Type & Content (\%) & Aid (\%) & $\mathbf{( 1 / 1 0} \mathbf{~ m m})$ & $\mathbf{( 1 / 1 0 ~ m m )}$ & Class \\
\hline Li-PAO & & 9.7 & 0 & 279 & 283 & 2 \\
Li-HOSO & \multirow{2}{*}{ Lithium hydroxy- stearate } & 15.9 & 0 & 264 & 272 & 2 \\
Li-OCT & & 13.7 & 0 & 279 & 294 & 2 \\
Li-TMPO & & 20.7 & 0 & 264 & 279 & 2 \\
\hline Ca-PAO & & 18.8 & 0 & 283 & 287 & 2 \\
Ca-HOSO & Calcium hydroxy- stearate & 17.6 & 0 & 272 & 279 & 2 \\
Ca-OCT & & 15.7 & 0 & 253 & 272 & 2 \\
Ca-TMPO & & 13.7 & 0 & 260 & 272 & 2 \\
\hline BT-PAO & & 28.1 & 1.07 & 249 & 279 & 2 \\
BT-HOSO & Bentonite & 15.8 & 1.12 & 257 & 268 & 2 \\
BT-OCT & & 24.0 & 1.18 & 242 & 279 & 2 \\
BT-TMPO & & 10.3 & 1.09 & 269 & 280 & 2 \\
\hline HDS-PAO & & 13.9 & 1.43 & 238 & 268 & 2 \\
HDS-HOSO & Highly dispersed silica acid & 11.4 & 0.89 & 264 & 287 & 2 \\
HDS-OCT & & 14.1 & 2.73 & 249 & 287 & 2 \\
HDS-TMPO & & &
\end{tabular}

${ }^{a} \mathrm{Pu}$ and Pw: unworked and worked penetration according to DIN ISO2137; ${ }^{\mathrm{b}}$ NLGI, National Lubricating Grease Institute; PAO, polyalpha olefin; HOSO, high-oleic sunflower oil; OCT, octyldodecyl isostearate; TMPO, trimethylolpropane trioleate. 


\subsection{Polarity Measurements}

In the literature, different ways to acquire the relative polarity of oils are suggested [17-22]. Interfacial tension measurements, as described in [23], provide a simple, yet accurate way to estimate oil polarities and to relate them to each other. These measurements were performed using a K100 Ring tensiometer (Krüss GmbH Wissenschaftliche Laborgeräte, Hamburg, Germany). For preparation of these measurements a suitable amount of distilled water is filled into a measuring glass and, after complete emersion of a cleansed platinum-iridium (PtIr) ring, a sufficient amount of oil is added to the water forming an interfacial surface. The tension of which is measured by slowly deforming it with the PtIr ring. The force applied in this deformation process is monitored and the deformation is continued until reaching a maximum value. This value is converted into the interfacial tension. Results of interfacial tension measurements are interpreted into relative polarities by ascribing high values of interfacial tensions between oil and water to low oil polarities.

\subsection{Rheological Tests}

Rotational rheological tests are usually performed to monitor the shear stress response over time at constant shear rates. The typical grease behavior in such rotational tests with constant shear rates, as described in $[24,25]$, shows a peak of maximum shear stress, which is related to the elastic response of the grease structure. This peak is followed by a steady decline as a result of the structural degradation of the grease and its consequent loss of viscosity. Subsequently, the shear stress responds with a convergent approximation to a limiting stable shear stress representing an equilibrium between shearinduced microstructural remaining stability and the applied shear rate [24-26]. The rheological investigations of the aforementioned greases were carried out on the modular compact, controlled-stress rheometer MCR300 (Anton Paar, Ostfildern, Germany). Rotational tests were performed using a plate-plate system with a diameter of $25 \mathrm{~mm}$, applying a constant shear rate of $1 \mathrm{~s}^{-1}$. All samples were given enough time to relax from the shearing stress during the closing of the gap and to adjust to temperature settings before the measurement was started. The measuring time was set to $2000 \mathrm{~s}$ which at $1 \mathrm{~s}^{-1}$ is enough time to reach the aforementioned limiting stable shear stress. All tests were performed at different temperatures $\left(-10{ }^{\circ} \mathrm{C}, 25^{\circ} \mathrm{C}, 40{ }^{\circ} \mathrm{C}\right.$ and $\left.80{ }^{\circ} \mathrm{C}\right)$. Every measurement for each of the 16 grease samples was repeated three times for each temperature.

\subsection{Atomic Force Microscopy (AFM) Observations}

In this study atomic force microscopy (AFM) offers a substantial advantage compared to other microscanning imaging methods, such as SEM, since the thickeners of the investigated greases do not need to be isolated, for instance by oil removing with a chemical solvent. Prior to the imaging process the greases are heated to temperature conditions below the dropping point of the respective grease for just a short period of time. Subsequently, they are cooled down forming an even surface, which can be scanned on the micro scale. Thus prepared, the samples are placed under the scanning probe of the AFM (Digital Instruments, Veeco Metrology Group Inc., Santa Barbara, CA, USA), which subsequently scans the grease surface mechanically in the non-contact mode. In this scanning mode the AFM tip oscillates at a frequency close to resonance while scanning the surface to be investigated. Van 
der Waals forces, and any other forces ranging far enough out of the surface, will attract the cantilever and deflect it, thus changing its oscillation frequency. This phase shift and the resulting amplitude are interpreted into an image of the surface.

\section{Results and Discussion}

\subsection{Polarity}

The interfacial tensions between all investigated base oils and distilled water were measured ten times repeatedly within a time period of $20 \mathrm{~min}$ in the above-described manner. Averaged results of these measurements are displayed in Table 2.

Table 2. Interfacial tensions measured between all investigated base oils and distilled water.

\begin{tabular}{ccccc}
\hline Base oil & PAO & HOSO & OCT & TMPO \\
\hline Interfacial tension $(\mathrm{mN} / \mathrm{m})$ & 20.0 & 9.0 & 23.0 & 7.5 \\
\hline
\end{tabular}

PAO, polyalpha olefin; HOSO, high-oleic sunflower oil; OCT, octyldodecyl isostearate; TMPO, trimethylolpropane trioleate.

According to the valid interpretation of interfacial tension measurements, these values result in a relative ranking of polarities of the given base oils in the following order: OCT $\approx$ PAO $\ll$ HOSO $\approx$ TMPO. These results are substantiated by the molecular structure of the investigated oleochemicals. Both, HOSO and TMPO are constituted of mainly triglyceride esters known to exhibit high electronegativities, which evoke a higher propensity to create permanent dipoles. However, this is not the case for PAO and especially OCT, which mainly consists of monoglycerides.

\subsection{Energy Density in Transient Flow}

As previously detailed [24,25,27-30], grease structure degradation process in transient flow tests may be described through the shear stress decay over time, from the stress overshoot, $\tau_{\max }$, representative of the initial elastic deformational process, to the remaining shear stress after completion of the degradation process, i.e., the steady-state value, $\tau_{\text {lim. Kuhn }}[30]$ further concludes that the integration of the $\tau(t)$ curve for any given period of time is to be interpreted as energy expenditure per stressed grease volume, namely the rheological energy density, which is expressed by:

$$
e_{\text {rheo-rot }}=\dot{\gamma} \int_{0}^{\zeta} \tau(t) d t
$$

where $\dot{\gamma}\left(s^{-1}\right)$ is the given constant shear rate applied and $\zeta(s)$ is the evaluation time period.

In previous studies [31], the energy density approach was used to interpret wear as a direct consequence of friction, so that the definition of wear is expanded and liberated from the limitation of the sole solid state unto the introduction of liquid state wear, meaning the degradation processes and the production of irreversibility in the separating lubricant film as a reaction to the applied frictional energy. Consequently, friction may be defined as an "irreversible process, which leads to the transformation and accumulation of mechanical energy accompanied by the production of entropy" 
(see [31] on page 32). Therefore, the aforementioned degradation process in rotational tests may be expressed as rheological wear.

Figure 1 shows different transient flow curves for selected Li-grease samples formulated with all examined base oils. In order to obtain values of rheological energy densities erheo-rot, as defined in Equation (1), the monitored development of shear stress over time was numerically integrated within bounds corresponding to $\tau_{\max }$ and $\tau_{\text {lim, }}$, respectively, as limiting time constraints. Results of these integrations of tests performed in exemplary temperature conditions of $-10{ }^{\circ} \mathrm{C}$ and $40{ }^{\circ} \mathrm{C}$ are displayed in Figures 2 and 3 with greases arranged in groups of thickeners. For the purpose of a better discernibility, the values were converted into $\mathrm{mJ} / \mathrm{mm}^{3}$ and displayed in linear scale.

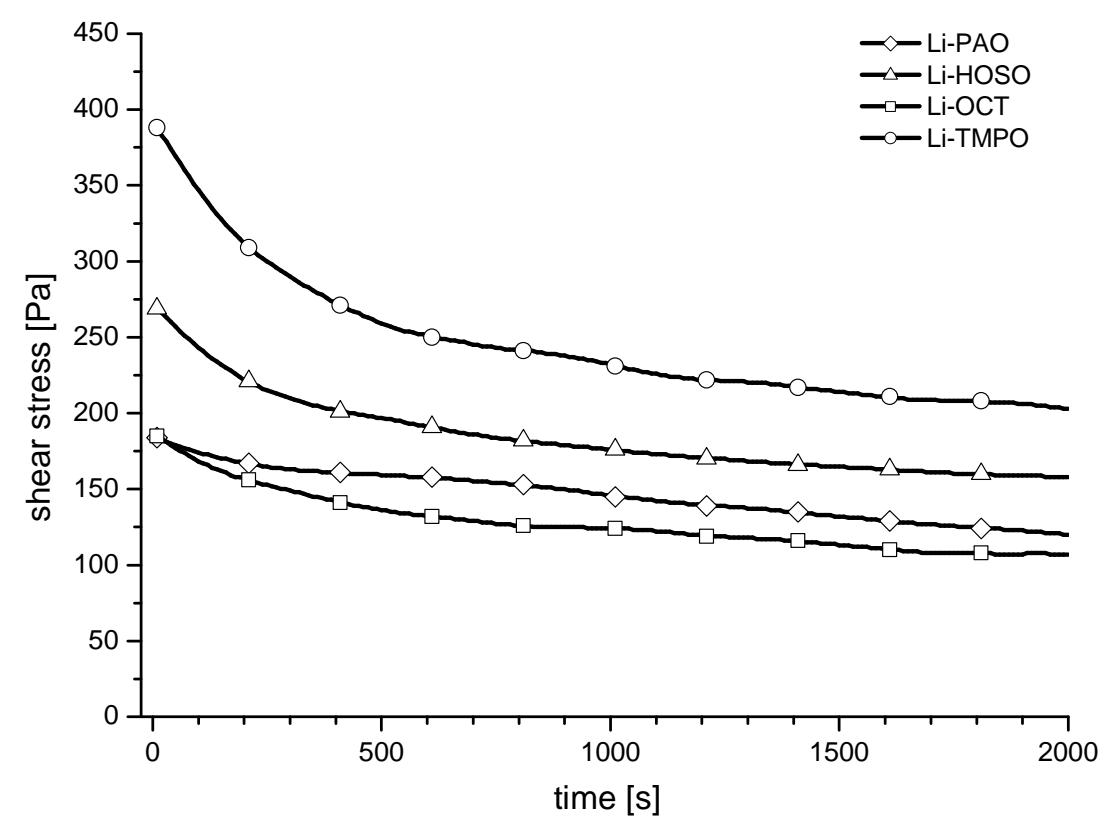

Figure 1. Transient flow curves of selected Li-grease samples determined at $40{ }^{\circ} \mathrm{C}$.

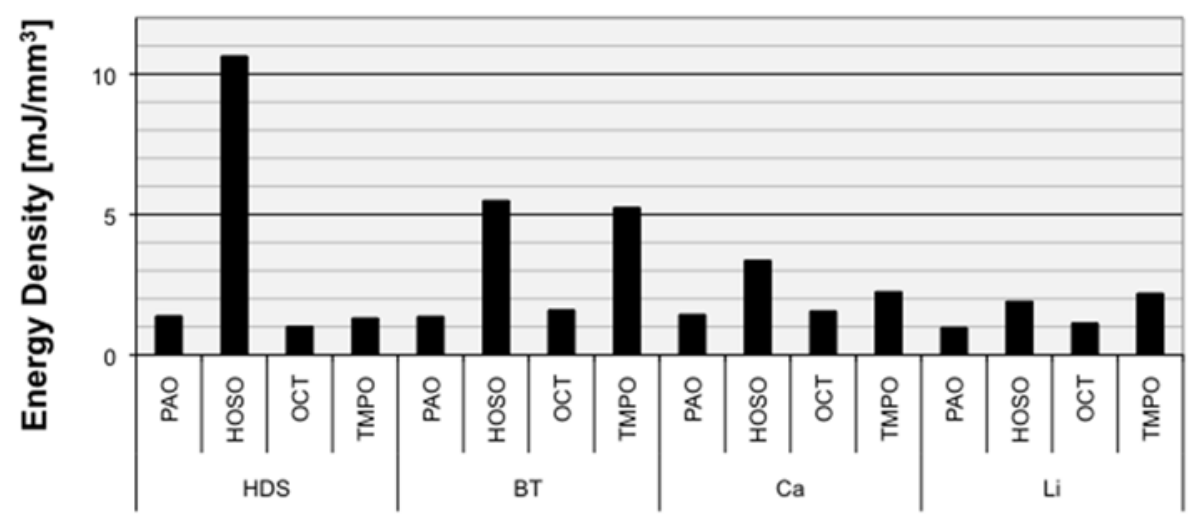

Grease Composition

Figure 2. Energy densities (e erheo-rot ) determined by integration of shear stress over time at $-10^{\circ} \mathrm{C}$. 


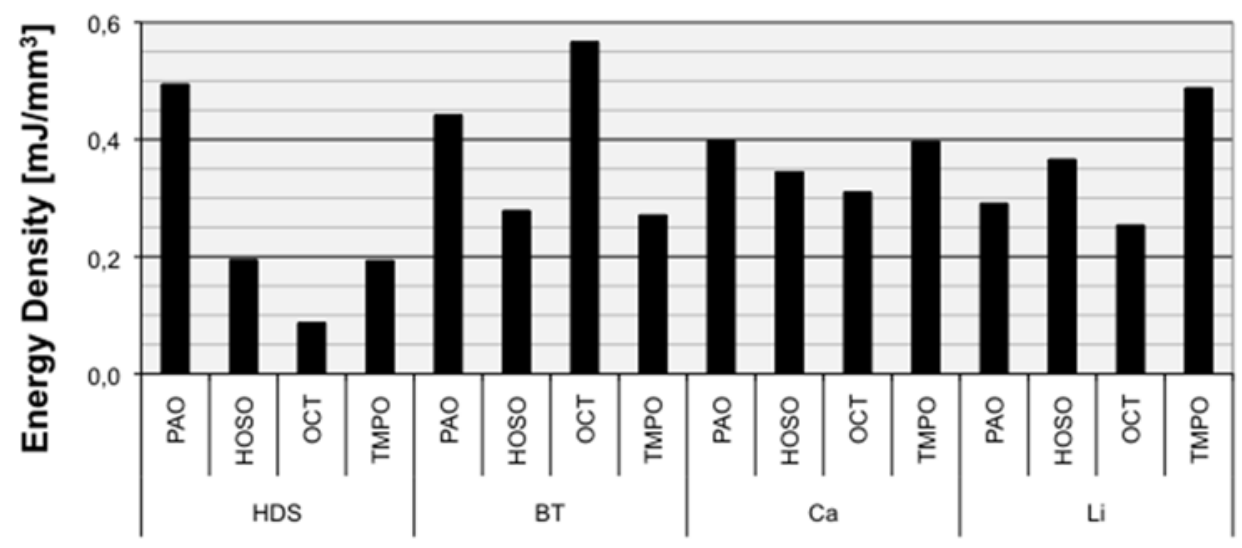

Grease Composition

Figure 3. Energy densities (erheo-rot) determined by integration of shear stress over time at $40{ }^{\circ} \mathrm{C}$.

The diagram in Figure 2 clearly depicts a good correlation between energy densities and base oil polarities. All greases based on the highly-polar oils, HOSO and TMPO, show a higher energy density within their respective thickener group than those based on the low-polarity oils, PAO and OCT. The only exception to this rule is the HDS-TMPO system, which exhibits a value of erheo-rot lower than expected. However, this result does not constitute an outlier because it is physically explicable by some effects which occurred only at very low temperature conditions. Thus, in all tests at $-10{ }^{\circ} \mathrm{C}$, it turned into solid matter, which was expelled from the measuring plate-plate gap at around $100 \mathrm{~s}$. So the true value of energy density of HDS-TMPO, although not detected, must be higher than displayed in the diagram. The difference of energy densities between greases formulated with high-polarity and low-polarity oils, respectively, is most apparent in the group of BT-greases $(72.4 \%)$, followed by Li-greases (48.7\%) and Ca-greases (46.5\%). If ignoring the value obtained for the HDS-TMPO system, the average difference of energy densities between high and low polarity greases rise up to $88.6 \%$ within the group of HDS-thickened greases.

The diagram of energy densities determined at $40{ }^{\circ} \mathrm{C}$ displayed in Figure 3 does not show this distinct differentiation between greases based on high and low polarity oils for all grease systems. Only Li-thickened greases reveal such behavior. Most interestingly the effect is reversed in the BT-thickened greases, with low polarity oils PAO and OCT now evoking higher energy densities in the greases.

As previously reported in [32], compositional variables are the most influencing factors affecting the rheology of greases. Particularly in this investigation, the nature of the different components (base oil and thickener), with individual solid or liquid characteristics, and concentrations are considered. The base oil viscosity and especially the percentage of thickener exert an influence on the structural degradation. The base oil viscosities do not differ significantly from each other in the given grease systems. The situation is different, however, with the percentage of thickener as shown in Table 1. These values reveal a polarity dependence of thickener percentage for all clay-thickened greases and for the Li-grease systems to fit a desired NLGI (National Lubricating Grease Institute) class 2. This influence is made manifest by a higher thickener ratio needed for highly polar oils than for low polarity oils. Ca-greases, on the other hand, do not show this dependence, probably due to the presence of the double charged cation. This influence most severely comes into effect within the groups of BT and 
HDS greases. In order to understand the underlying mechanisms of this effect one must consider the structure and composition of clay thickeners.

Bentonite, e.g., is a clay-based thickener extracted from clays of volcanic ashes with montmorillonite, a triple-layer particle structure phyllosilicate, as main constituent. Montmorillonite itself chemically consists of $60 \% \mathrm{SiO}_{2}$ and $20 \% \mathrm{Al}_{2} \mathrm{O}_{3}$ and contains several other metal ions stored in the layer lattice [33]. Substitution of these ions by quaternary ammonium salts results in organophillic modification, which enables thickening processes of petrochemical as well as oleochemical oils [33-36]. As a result of this modification the surface of the montmorillonite particles is activated with hydrocarbon alkyls of different chain lengths. The length and polarity of these chains influences the affinity for oils according to their polarity. In the thickening process intense shearing is needed in the process of blending the base oil with the organically modified bentonite in order to break up the plate layer aggregates and completely wet all surfaces. Addition of other chemical activators (carbonates, glycols, ketones, alcohols, and especially water) is needed to overcome adhesion forces between the particles and enable the thickening process. During the latter, the particles form a three-dimensional stable network, which physically traps the base oil [33,37]. According to Goerz [33], the hydrocarbon alkyl-chain rests highly influence the bonding character and the consequent thickening behavior in BT greases. For this reason highly polar oils more naturally attach to these hydrocarbon molecule chain rests in organosilicon particles of BT greases than low polarity oils. Thus, they interfere with, and partially prevent, the formation of the BT matrix. Therefore, it is stressed that tailor-made organophyllic modifiers should be used for each prevailing oil polarity. This fact was not considered in the formulation of the investigated model greases. For this reason the use of the same BT thickener type, containing equal ammonium ions with similar hydrocarbon alkyl-chains, must result in individual thickener percentages for all base oils.

As can be seen in Table 1, the formulation of HDS greases is also subjected to polarity influences. Due to its main constituent, $\mathrm{SiO}_{2}$, HDS-thickener, like BT is regarded as a clay thickener. In the production process, quartz sands are firstly enriched with chlorine resulting in $\mathrm{SiCl}_{4}$, which is then pyrolytically cracked in oxyhydrogen-reactive flame hydrolysis resulting in $\mathrm{SiO}_{2}$ primary particles consisting of tetrahedral $\mathrm{SiO}_{4}$ connected via siloxane bridges and outstanding silanol groups. These primary particles link together in the flame reaction and form flaky chain aggregates with a very high specific surface (up to $400 \mathrm{~m}^{2} / \mathrm{g}$ ). The silanol groups of the primary particles enable hydrogen bonds to organosilicon compounds in the hydrophobization process and to the hydrocarbon chains of surrounding base oils in the thickening process. Through the latter, a three-dimensional network structure is formed that provides mechanical stability [33]. It is observed that highly polar oils show a higher tendency to dock with Si-OH groups, protruding from primary particles of HDS greases, than low-polarity oils. This causes a disturbance of the formation of the three-dimensional network and generally results in higher percentages of thickener needed to thicken highly polar oils with equal mechanical stability.

So far, two different polarity-related effects on greases and their characteristics have emerged as illustrated in Figure 4. In this chart curved filled arrows represent all direct influences whilst slim straight arrows represent possible mutilations of direct influences. On the one hand, there is a direct influence on the base oil/thickener ratio even in the formulation of the greases, as discussed earlier. On the other hand the structural breakdown of greases under transient shear is also directly affected by 
base oil polarities, as has been shown by means of the energetic evaluation of the transient shear results. This double effect makes it exceedingly difficult to reveal the exclusive influence of the base oil polarity on mechanical structural breakdown because this degradation is also essentially influenced by the thickener concentration. In order to disclose the exclusive influence of the base oil polarity on mechanical structural degradation it is necessary to establish measurement conditions, which solely reveal the same by keeping constant all other influencing factors. The bond forces evoked and influenced by base oil polarities are mainly Keesom forces, as discussed in the introduction of this article. They are the strongest secondary valence bond forces, hence, in the context of adjustment of measuring conditions, it seems plausible to find conditions which solely mutilate or even eliminate Keesom forces. It is well known that the formation of Keesom forces is highly temperature-dependent [11]. Through atomic scale oscillation evoked by a temperature increase, they are intensively reduced. In other words, the higher the medium temperature during the test, the lower the Keesom forces in the grease microstructure are. At low temperature conditions, however, Keesom forces are maximized. For this reason the above-described temperature range from $-10{ }^{\circ} \mathrm{C}$ up to $80{ }^{\circ} \mathrm{C}$ was chosen and polarity influences were isolated from other effects, such as percentage of thickener content. This is illustrated by the slim arrows in Figure 4 pointing to the bold filled arrows representing a mutilation of these direct influences. This way Figure 4 makes clear that the temperature only disturbs the direct influence of base oil polarity on the structural breakdown. In reality the temperature could also marginally mutilate the influence of base oil polarities on the thickener concentration. This influence, as inherently limited, is only exerted in the moment of grease production and loses its effect after completion of the production. Moreover the temperature does not mutilate the influence of the base oil/thickener ratio on the structural breakdown in transient shear.

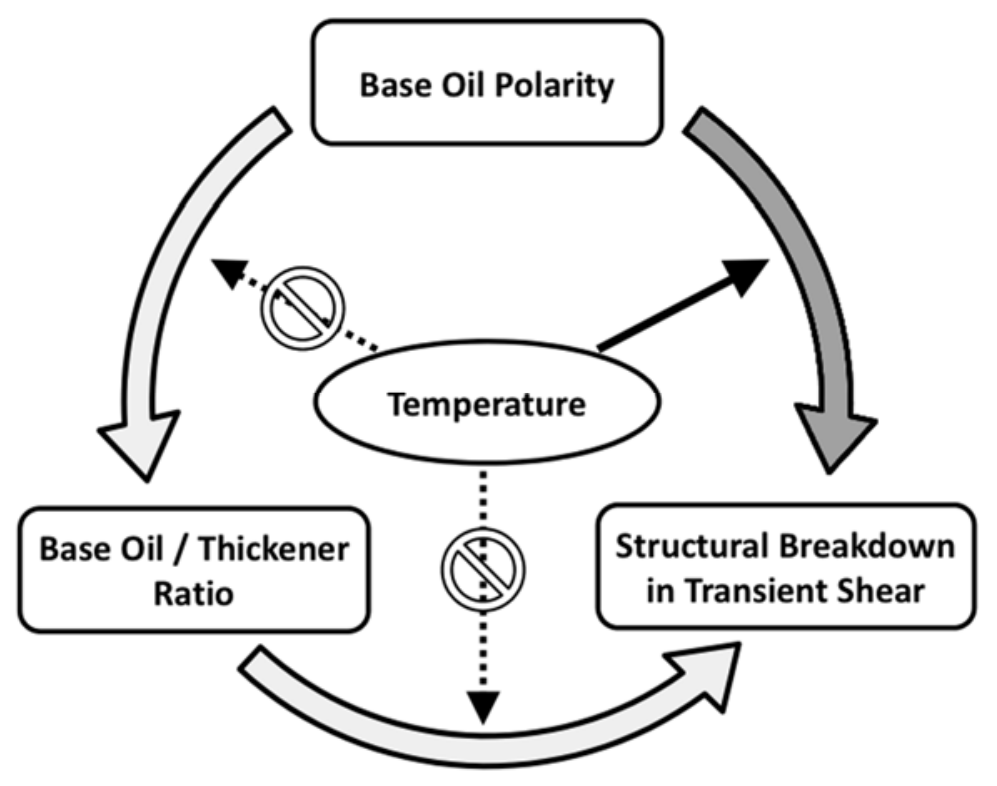

Figure 4. Scheme considering the different polarity influences on structural breakdown under transient shear. Note: Bold curved arrows represent direct influences, slim straight arrows (dotted and solid) represent possible mutilations of direct influences. 
For further discussion of the investigation of temperature influences, all energy densities analytically determined by integration according to Equation (1) are plotted as a function of temperature in the complete region of all measurement points. Figure 5 collects these values of energy densities for all greases studied. In this data plot the grease systems with highly polar oils, TMPO, and HOSO, are color-coded in black solid lines with different line tags for the respective groups of thickeners and base oils. All curves of greases based on low-polarity oils are color-coded with gray solid lines without any information on the thickener or base oil. This figure clearly shows that energy densities determined at temperatures higher than $25^{\circ} \mathrm{C}$ all quite much resemble. The largest deviation from a linear trend mainly occurs in measurements performed at $-10{ }^{\circ} \mathrm{C}$. This deviation from a linear trend highly depends on the base oil polarity. While all energy densities determined for greases based on low-polarity oils stay within a narrow fluctuation band even at $-10{ }^{\circ} \mathrm{C}$ the greases based on highly polar oils do not show this behavior. In fact they even result in curves that are best fitted by an exponential approximation. The largest deviations from a linear trend are found for the grease systems formulated with clay thickeners. As expected, HDS-TMPO is an exception to this rule for the priordiscussed reasons. The greases formulated with metal soaps and highly polar oils measured at $-10{ }^{\circ} \mathrm{C}$ only slightly but still clearly result in higher energy densities than all other greases based on low polarity oils.

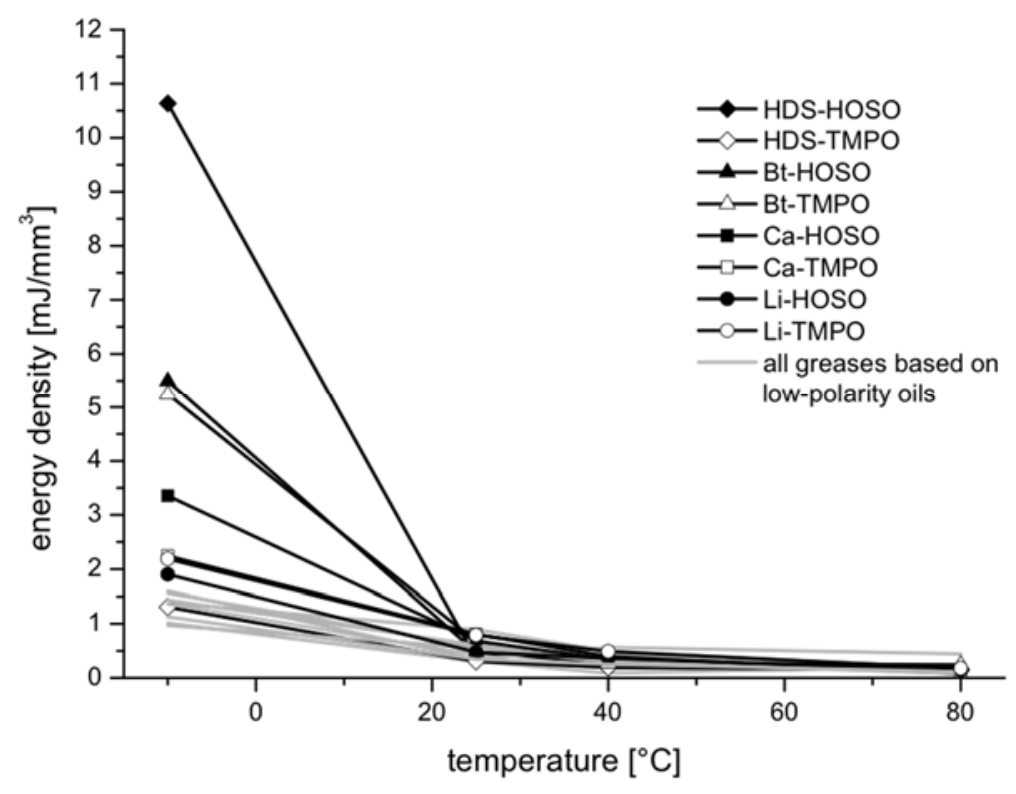

Figure 5. Energy densities (erheo-rot) vs. temperature plot.

This apparent exponential temperature dependent behavior of energy densities leads to the assumption that it may follow an Arrhenius type fitting. Adaption of the Arrhenius-type fitting to the here-investigated physical process of temperature dependent degradation under transient shear leads to:

$$
e_{\text {rheo-rot }}=A e^{\left(\frac{E \mathrm{a}}{R T}\right)}
$$

where $T(\mathrm{~K})$ represents the temperature, $\mathrm{R}$ represents the universal gas constant $\left(8.314 \mathrm{~J} \cdot \mathrm{K}^{-1} \cdot \mathrm{mol}^{-1}\right)$, $\mathrm{A}$ is a dimensionless pre-exponential factor and $E_{\mathrm{a}}\left(\mathrm{J} \cdot \mathrm{mol}^{-1}\right)$ is an activation energy which, in this case, may have a physical meaning of the energy input necessary to produce structural degradation in the 
grease sample under transient shear. Plotting the results of Arrhenius fits in logarithmic scale over the reciprocal temperature reveals the ratio of $E_{\mathrm{a}}$ and $R$ as the slope of the function. Figure 6 depicts the original data along with the corresponding Arrhenius type fits in the mentioned scales of some selected highly-polar and non-polar greases. Excepting for HDS-HOSO system, reasonably good fitting was obtained considering only the four temperatures analyzed. This figure, directly confronting both polarity groups, also shows a clear distinction between highly polar and low-polarity greases. It appears that the highly polar oil-based grease systems require a larger portion of activation energy in order to produce structural degradation in the grease samples under transient shear. This presumption is supported with the data shown in Figure 7 where all values of activation energy $E_{a}$ are displayed in groups of thickeners. In all of these groups a polarity influence more or less severely affects the activation energy, resulting in higher activation energies necessary to evoke structural degradation in transient shear with greases based on highly-polar oils than with those based on low-polarity oils.

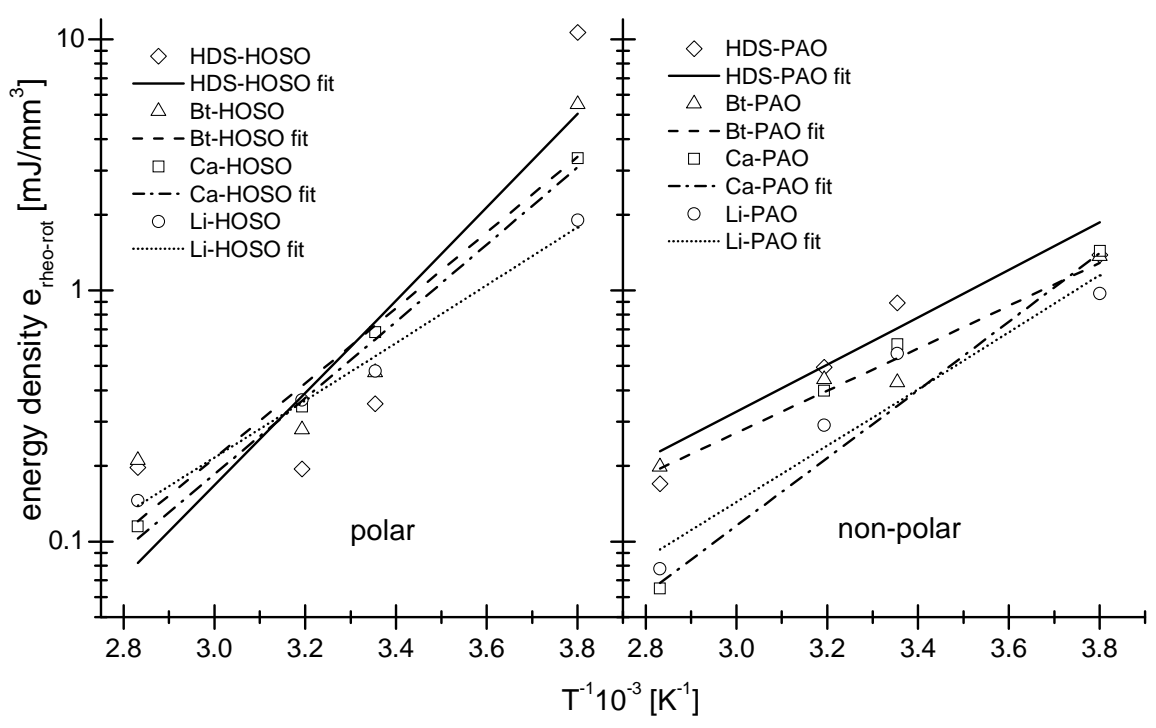

Figure 6. Original data and Arrhenius fits of greases based on highly polar high-oleic sunflower oil (HOSO) and non-polar polyalpha olefin (PAO). HDS, highly dispersed silica acid; Bt, bentonite; Ca, calcium; Li, lithium.

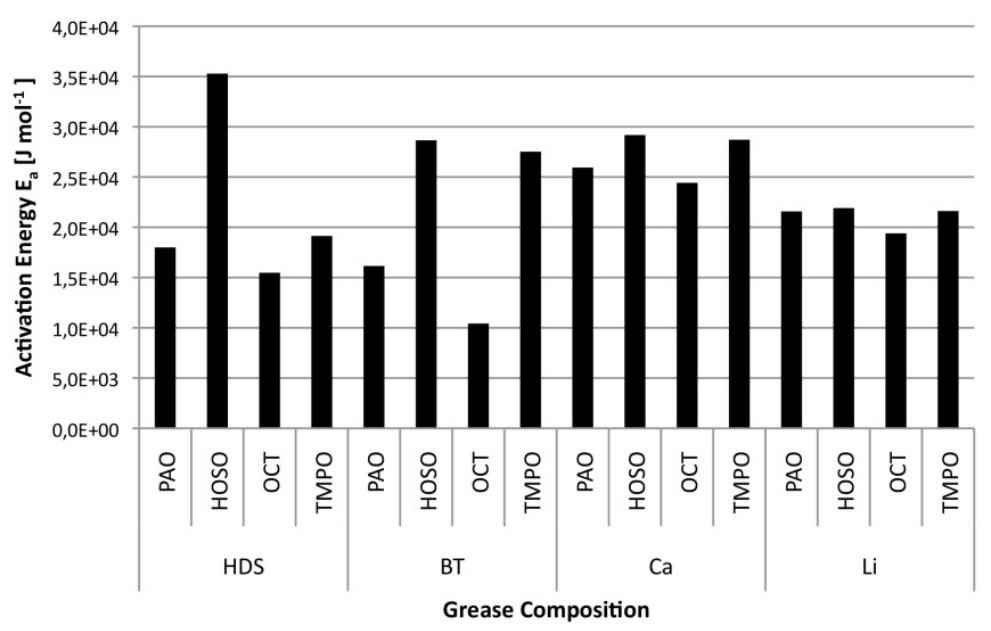

Figure 7. Activation energy, $E_{\mathrm{a}}$, in transient shear flow. 
This effect results, most significantly, in larger differences between greases based on high and low-polarity oils within the groups of clay thickened greases HDS (38.5\%) and BT (52.7\%). HDS-TMPO, for explained reasons, is presumed to have resulted in much higher activation energy than displayed by the found data. A polarity influence is also found in both soap-thickened grease systems although its effect results in smaller differences between the activation energies of high and low polarity greases - $\mathrm{Ca}(13.0 \%)$ and $\mathrm{Li}(5.9 \%)$. This effect may be explained by the stronger bond forces evoked between the thickener matrix and the highly polar oils surrounding it. It is presumed that the clay thickeners, which all required more activation energy to commence the structural breakdown in combination with highly polar oils, more naturally interact with the same. Low-polarity base oils consequently result in less activation energy needed in the process of structural breakdown. It is generally noted that clay thickeners react much more sensitively to a change of oil polarity in lubricating greases. Reasons for this effect are found in the microstructure and the physical mode of action of all investigated thickeners as will be discussed in the next section.

\subsection{Atomic Force Microscopy (AFM)}

In order to elucidate the above-discussed behavior, one needs to understand the physical functionality of the investigated thickener systems at the molecular as well as the macro level. This especially applies if considering that only the synergy and interaction of the different grease ingredients on the mentioned levels cause the overall transient flow response. Figure 8 displays selected AFM micrographs of four of the greases investigated in this study.

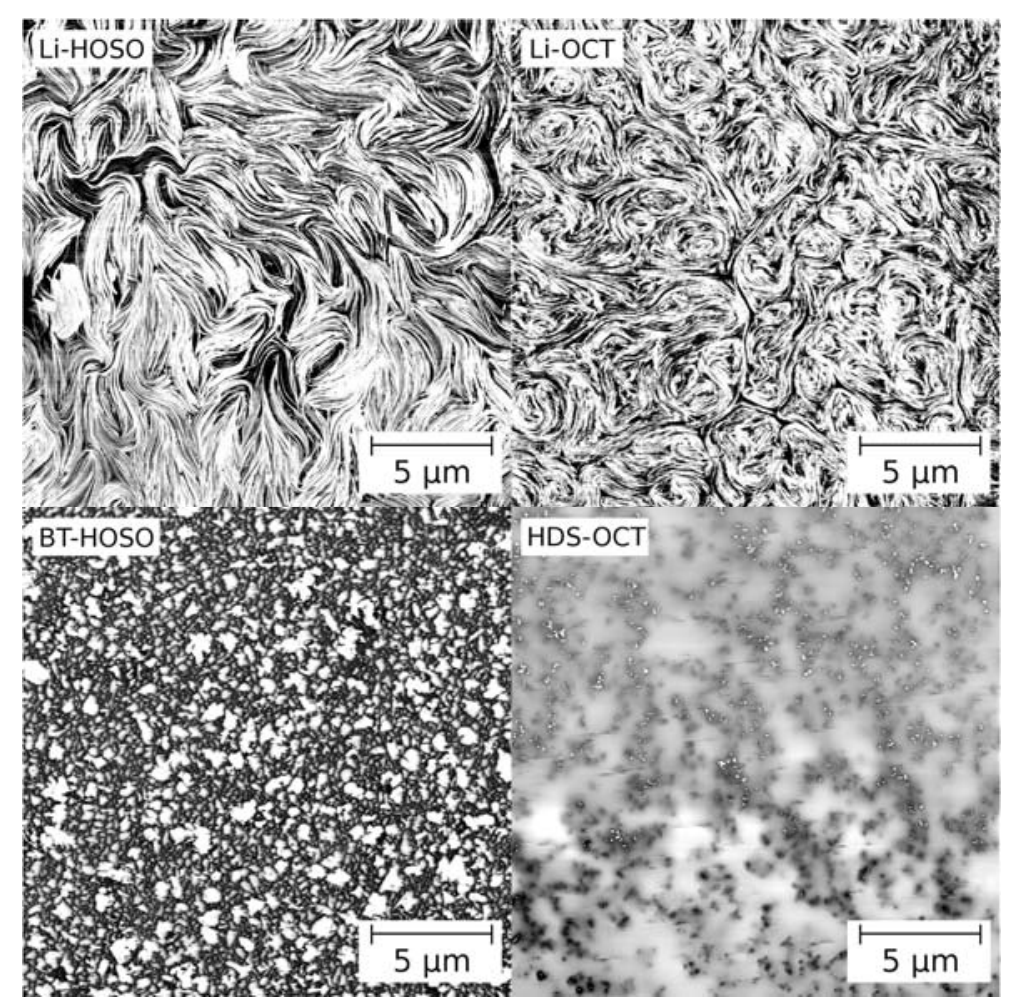

Figure 8. Atomic Force Microscopy (AFM) micrographs of four examined sample greases (image width: $20 \times 20 \mu \mathrm{m}$ ). Li, lithium; HOSO, highly polar high-oleic sunflower oil; OCT, octyldodecyl isostearate; BT, bentonite; HDS, highly dispersed silica acid. 
The thickeners of the greases appear as bright to white areas in the partial images of Figure 8 . The brighter an area appears in the AFM image the more rigid is the scanned part of the specimen. Dark areas in the AFM image analogously represent soft or even liquid parts representing the enclosed base oil. It should be mentioned that the rigidities of the displayed AFM images in Figure 8 are not to be compared to each other because each partial image was manually normalized to picture the full spectrum of gray scales before they were put together. Still, the structure and the composition of the grease thickeners appear very clearly. The two split images of the Li greases very clearly depict the fibrillar structure of the thickener with single, partially interwoven and entangled loops of different size. The two bottom split images of the clay-thickened greases very clearly display clusters of spherical or platelet solid particles. In the case of BT-HOSO one not only notices the size of these platelets but also how densely and multi directionally they are arranged. The HDS-OCT, on the other hand, displays only a few very small solid particle aggregates distributed evenly in the visible superficial layer. This is attributed to the very small size of the primary particles of HDS. They form a rather rigid system if mixed thoroughly as marked in grayish areas. However, if they agglutinate, as marked in bright spots, the thickener particles lose their ability to tie the base oil as shown in the dark edges surrounding each aggregate.

Figure 8 allows to elucidate the physical mechanism acting in the above-discussed transient shear flow as well as the mixing ratio of the greases. Hence, a lubricating grease based on a chemically-bound metallic soap with all its fibrillar, interwoven, and entangled loops is expected to respond differently to changing oil polarities than a clay-grease with its micro platelet structure. This occurs although both thickening systems only physically interact with the surrounding base oil and with each other. Figure 8 also shows that the base oil polarity may very well influence the type and length of Li-soap fibers. Longer and less entangled fibers with less loops per examined unit of volume result from the combination with highly polar HOSO as compared to the low polarity base oil OCT. This circumstance permits the deduction that base oil polarities in combination with Li thickeners may exert an influence on the thickener/base oil ratio as shown in Table 1. In addition, the fact that base oil polarities only marginally affect the activation energy needed for structural breakdown in transient shear, which might also be labeled a thickener-share-adjusted degradation behavior, leads to the presumption that they exert their influence on Li greases mainly once-during the formulation of the grease. As soon as the Li soap is completely formulated, the indirect influence of base oil polarities on the transient flow response depends on the length, type and, rate of entanglement as well as on the ratio of soap fibers.

For technical reasons it was not possible to obtain accurate AFM micrographs of the examined Ca greases. Hence, the response of their microstructure to alternating polarities cannot be fully elucidated. Due to the above-discussed polarity-independent constant thickener ratio (see Table 1) it is presumed, however, that the structure of $\mathrm{Ca}$ greases is independent of oil polarity to a large degree. In combination with the relatively high polarity difference in the thickener-share-adjusted evaluation of grease degradation by means of activation energy it is presumed that the structural breakdown of Ca-thickened greases is mainly subject to polarity influences.

As previously mentioned, the platelets of the clay particles are considerably smaller than the fibers of the soap greases. They can only physically interact with each other in the formation of the supporting matrix structure. The smaller the particles, the more bonding sites are available per 
considered volume unit. Therefore, it is presumed that they evoke greater polarity-dependent rheological shear stress responses in the grease. Furthermore, this is substantiated by the fact that the interaction of the solid body particles only occurs through the surrounding base oil in the formation of the support network. In other words, the base oil works as an adhesive between the particles. This is also the case for soap fibers, yet they are significantly longer and, thus, although naturally offering a greater target surface per fiber in this adhesion process, they offer less target surface per considered unit of grease volume. In addition, they are much more flexible than clay particles, and thus they offer a greater contact surface even in sliding produced by mechanical shearing. In summary, it is presumed that the rheologically-measured polarity-dependence of clay greases is evoked by the small size of clay particles, their solid body characteristics and its associated lack of flexibility. This would also elucidate why, with the activation energy, the clay-thickened grease systems HDS and BT showed greater polarity dependence than the $\mathrm{Li}$ and $\mathrm{Ca}$ soap-thickened greases.

\section{Conclusions}

An attempt has been made to disclose the exclusive polarity influence on the structural degradation in transient shear by means of Arrhenius type fitting of energy densities. This approach constitutes a thickener-share-adjusted consideration of polarity influences. In comparison of this approach to the sole evaluation of energy densities in transient shear, which is still vitiated by a thickener-share influence, one finds that the extend of influences resulting from the activation energy corresponds more to the energetic behavior in low temperature conditions $\left(-10^{\circ} \mathrm{C}\right)$. On the other hand, the pure thickener ratio is best reflected by the energetic evaluation at high temperatures $\left(40{ }^{\circ} \mathrm{C}\right.$ and higher). This is not surprising because the Keesom-forces, and consequently the influence of polarity, diminish with higher temperatures which, in turn, results in a predomination of the influence of thickener ratio. The fact that the energetic evaluation at low temperatures more corresponds to the results found with the activation energy indicates that in it polarity influences clearly predominate.

The results of this study demonstrate that there are really two different base oil polarity influences on the rheologically-measured structural degradation of lubricating greases in transient shear flow. On the one hand, a polarity-dependent influence on the thickener/base oil ratio was detected. This, in turn, exerts a unique influence on some rheological characteristics. On the other hand, the base oil polarity, considered alone, does also influence the rheological grease behavior in transient shear. This was found with an Arrhenius type approximation of the temperature-dependent function of energy densities in combination with the temperature-dependent formation of Keesom forces. Depending on the applied thickener, these tendencies show different intensities. In a thickener-share-adjusted interpretation of the energy density temperature dependence by means of Arrhenius type fitting, it is found that, in fact, both polarity-induced influences are exerted in the clay-thickened systems. Within the group of clay-thickened greases, the highly polar oils increase not only the percentage of thickener but also the activation energy necessary to produce structural degradation. This double polarity influence, although not as severely, is also found in the group of Li soap-thickened greases. Here also, the thickener ratio and the level of activation energy needed for structural breakdown are affected by base oil polarities. In the Ca greases, however, there is no detectable influence on the thickener/base oil ratio, whilst there is a slight tendency pointing to a polarity dependence of activation energy in the mechanical degradation. 


\section{Author Contributions}

The text and figures in this manuscript were created by Martin Fiedler during the course of writing a $\mathrm{PhD}$ thesis at the University of Huelva. José M. Franco and Erik Kuhn contributed by supervising the experimental work and amending text passages and images. Rubén Sánchez, Concepción Valencia and Claudia S. Leopold provided a valuable technical and experimental contribution.

\section{Conflicts of Interest}

The authors declare no conflict of interest.

\section{References}

1. Lugt, P.M. A review on grease lubrication in rolling bearings. Tribol. Trans. 2009, 52, 470-480.

2. Venner, C.; van Zoelen, M.; Lugt, P. Thin layer flow and film decay modeling for grease lubricated rolling bearings. Tribol. Int. 2012, 47, 175-187.

3. Balan, C.; Franco, J.M. Influence of the geometry on the transient and steady flow of lubricating greases. Tribol. Trans. 2001, 44, 53-58.

4. Franco, J.M.; Delgado, M.A.; Valencia, C.; Sanchez, M.C.; Gallegos, C. Mixing rheometry for studying the manufacture of lubricating greases. Chem. Eng. Sci. 2005, 60, 2409-2418.

5. Delgado, M.A.; Valencia, C.; Sánchez, M.C; Franco, J.M; Gallegos, C. Thermorheological behaviour of a lithium lubricating grease. Tribol. Lett. 2006, 23, 47-54.

6. Martin-Alfonso, J.E.; Valencia, C.; Sanchez, M.C; Franco, J.M.; Gallegos, C. Development of new lubricating grease formulations using recycled LDPE as rheology modifier additive. Eur. Polym. J. 2007, 43, 139-149.

7. Delgado, M.A; Franco, J.M; Valencia, C.; Kuhn, E.; Gallegos, C. Transient shear flow of model lithium lubricating greases. Mech. Time Depend. Mater. 2009, 13, 63-80.

8. Wilson, B. Lubricants and functional fluids from renewable sources. Ind. Lubr. Tribol. 1998, 50, 6-15.

9. Cousseau, T.; Graça, B.; Campos, A.; Seabra, J. Friction and wear in thrust ball bearings lubricated with biodegradable greases. Proc. Inst. Mech. Eng. Part J J. Eng. Tribol. 2011, 225, $627-639$.

10. Lugscheider, E.; Bobzin, K. Wettability of PVD compound materials by lubricants. Surf. Coat. Technol. 2003, 165, 51-57.

11. Massmann, T.C. Wirkmechanismen additivierter Schmierstoffe in der Kaltumformung. Ph.D. Thesis, RWTH Aachen University, Aachen, Germany, 2007.

12. Owens, D.; Wendt, R. Estimation of the surface free energy of polymers. J. Appl. Polym. Sci. 1969, 13, 1741-1747.

13. Mortimer, C.; Müller, U. Chemie: Das Basiswissen der Chemie; Thieme Verlag: Stuttgart, Germany, 2003.

14. Fiedler, M.; Sanchez, R.; Kuhn, E.; Franco, J.M. Influence of oil polarity and material combination on the tribological response of greases formulated with biodegradable oils and bentonite and highly dispersed silica acid. Lubr. Sci. 2013, 25, 397-412. 
15. Zaki, N.; Litters, T. Investigation of low temperature behavior of lubricating greases by strain sweep rheometry-Effect thickener and base oil on viscoelastic properties at $-40{ }^{\circ} \mathrm{C}$. NLGI Spokesm. 2009, 72, 7-23.

16. Litters, T.; Koch, B. Einfluss der Basisölpolarität auf das viskoelastische Verhalten von Schmierfetten. In Fachtagung der GfT; Gesellschaft für Tribologie e.V.: Aachen, Germany, 2008. (In German)

17. Zainal, Z.; Isengard, H.D. Determination of total polar material in frying oil using accelerated solvent extraction. Lipid Technol. 2010, 22, 134-136.

18. Dobarganes, M.C.; Velasco, J.; Dieffenbacher, A. Determination of polar compounds, polymerized and oxidized triacylglycerols, and diacylglycerols in oils and fats. Pure Appl. Chem. 2000, 72, 1563-1575.

19. Knorn, B.; Göbel, R.; Franzke, C. Bestimmung der Polarität gesättigter Monoglyceride durch Gaschromatographie. Food Nahr. 1977, 21, 817-823. (In German)

20. Rouser, G.; Kritchevsky, G.; Galli, C.; Heller, D. Determination of polar lipids: Quantitative column and thin-layer chromatography. J. Am. Oil Chem. Soc. 1965, 42, 215-227.

21. Márquez-Ruiz, G.; Jorge, N.; Martín-Polvillo, M.; Dobarganes, M.C. Rapid, quantitative determination of polar compounds in fats and oils by solid-phase extraction and size-exclusion chromatography using monostearin as internal standard. J. Chromatogr. A 1996, 749, 55-60.

22. Ringard-Lefebvre, C.; Bochot, A.; Memisoglu, E.; Charon, D.; Duchêne, D.; Baszkin, A. Effect of spread amphiphilic $\beta$-cyclodextrins on interfacial properties of the oil/water system. Colloids Surf. B Biointerfaces 2002, 25, 109-117.

23. El-Mahrab-Robert, M.; Rosilio, V.; Bolzinger, M.A.; Chaminade, P.; Grossiord, J.L. Assessment of oil polarity: Comparison of evaluation methods. Int. J. Pharma. 2008, 348, 89-94.

24. Kuhn, E. Experimental grease investigations from an energy point of view. Ind. Lubr. Tribol. 1999, 51, 246-251.

25. Kuhn, E. Influence of the soap content of lubricating greases on the tribological process. Eurogrease 2007, 1, 7-11.

26. Delgado, M.; Franco, J.M.; Kuhn, E. Effect of rheological behaviour of lithium greases on the friction process. Ind. Lubr. Tribol. 2008, 60, 37-45.

27. Kuhn, E. An algorithm to estimate the friction energy of a grease lubricated contact. Ind. Lubr. Tribol. 2000, 52, 174-177.

28. Kuhn, E. Irreversible Eigenschaftsaenderung durch Reibungsbeanspruchung bei Schmierfetten-Rheologischer verschleiss. Tribologie und Schmierungstechnik 2006, 53, 27-29. (In German)

29. Kuhn, E. Description of the energy level of grease lubricated contacts. In Proceedings of the AITC-AIT, 5th International Conference of Tribology, Parma, Italy, 20-22 September 2006.

30. Kuhn, E. Zur Tribologie der Schmierfette; Expert Verlag: Renningen, Germany, 2009. (In German)

31. Kuhn, E. Der Schmierstoffverschleiß. Tribologie und Schmierungstechnik 2011, 58, 32-35. (In German)

32. Delgado, M.A.; Valencia, C.; Sanchez, M.C.; Franco, J.M.; Gallegos, C. Influence of soap concentration and oil viscosity on the rheology and microstructure of lubricating greases. Ind. Eng. Chemistry Res. 2006, 45, 1902-1910. 
33. Goerz, T. Gel-und Bentonitfette Zusammensetzung-Eigenschaften. Tribologie und Schmierungstechnik 2009, 56, 30-34. (In German)

34. Pogosyan, A.; Martirosyan, T. Tribological properties of bentonite thickener-containing greases. J. Frict. Wear 2008, 29, 205-209.

35. Chtourou, M.; Frikha, M.; Trabelsi, M. Modified smectitic Tunisian clays used in the formulation of high performance lubricating greases. Appl. Clay Sci. 2006, 32, 210-216.

36. Kohashi, H. Application of fatty acid esters for lubricating oil. In Proceedings of World Conference on Oleochemicals into the 21st Century, American Oil Chemists Society, Champaign, IL, USA, 21-25 January 1991.

37. Dresel, W. Biologically degradable lubricating greases based on industrial crops. Ind. Crops Prod. 1994, 2, 281-288.

(C) 2015 by the authors; licensee MDPI, Basel, Switzerland. This article is an open access article distributed under the terms and conditions of the Creative Commons Attribution license (http://creativecommons.org/licenses/by/4.0/). 